\title{
SOME REMARKS ON GRADIENT ESTIMATES FOR HEAT KERNELS
}

\author{
NICK DUNGEY
}

Received 27 September 2004; Accepted 1 March 2005

This paper is concerned with pointwise estimates for the gradient of the heat kernel $K_{t}$, $t>0$, of the Laplace operator on a Riemannian manifold $M$. Under standard assumptions on $M$, we show that $\nabla K_{t}$ satisfies Gaussian bounds if and only if it satisfies certain uniform estimates or estimates in $L^{p}$ for some $1 \leq p \leq \infty$. The proof is based on finite speed propagation for the wave equation, and extends to a more general setting. We also prove that Gaussian bounds on $\nabla K_{t}$ are stable under surjective, submersive mappings between manifolds which preserve the Laplacians. As applications, we obtain gradient estimates on covering manifolds and on homogeneous spaces of Lie groups of polynomial growth and boundedness of Riesz transform operators.

Copyright (c) 2006 Nick Dungey. This is an open access article distributed under the Creative Commons Attribution License, which permits unrestricted use, distribution, and reproduction in any medium, provided the original work is properly cited.

\section{Introduction}

Let $M$ be a connected, complete Riemannian manifold. Write $d(x, y)$ for the Riemannian distance between $x, y \in M$ and $d x$ for the Riemannian measure. We set $B(x, r)=$ $\{y \in M: d(x, y)<r\}$ and $V(x, r):=d x(B(x, r))$ for $r>0$. Let $H=-\operatorname{div} \nabla$ be the positive Laplace operator, where div and $\nabla$ are respectively the Riemannian divergence and gradient on $M$. We consider the heat semigroup $S_{t}=e^{-t H}, t \geq 0$, which acts in $L^{p}=L^{p}(M ; d x)$, $1 \leq p \leq \infty$, and the heat kernel $K_{t}(x, y)$ defined for $t>0, x, y \in M$. We will assume the volume doubling property, that is, there exists $c>0$ such that

$$
V(x, 2 r) \leq c V(x, r)
$$

for all $x \in M$ and $r>0$, and the Gaussian heat kernel upper bound

$$
K_{t}(x, y) \leq c_{\omega} V\left(y, t^{1 / 2}\right)^{-1} e^{-\omega d(x, y)^{2} / t}
$$

Hindawi Publishing Corporation

Abstract and Applied Analysis

Volume 2006, Article ID 73020, Pages 1-10

DOI 10.1155/AAA/2006/73020 
2 Some remarks on gradient estimates for heat kernels

for all $\omega \in(0,1 / 4), t>0$, and $x, y \in M$, where $c_{\omega}$ is a constant depending on $\omega$. The conjunction of assumptions (1.1) and (1.2) is well understood, and it is known that (1.2) is a consequence of the on-diagonal estimate $K_{t}(x, x) \leq c V\left(x, t^{1 / 2}\right)^{-1}, t>0, x \in M$ (see, e.g., $[7,10]$ and references therein).

In this paper we study certain gradient estimates for $K_{t}$, which may or may not hold under the above assumptions. Such estimates have intrinsic interest as regularity properties of the heat kernel, but are also closely connected with the boundedness in $L^{p}(M)$ of Riesz transform operators (see, e.g., [2]).

Adopt the convention that $\nabla K_{t}(x, y)=\nabla_{x} K_{t}(x, y)$ denotes the gradient with respect to the first variable of the two variable kernel $K_{t}(\cdot, \cdot)$, and consider the following conditions.

Condition (I). There exist $c, b>0$ such that

$$
\left|\nabla K_{t}(x, y)\right| \leq c t^{-1 / 2} V\left(y, t^{1 / 2}\right)^{-1} e^{-b d(x, y)^{2} / t}
$$

for all $t>0, x, y \in M$ (here, $|\cdot|$ denotes the Riemannian length of tangent vectors).

Apparently weaker than Condition (I) is

Condition (II). There is $c>0$ such that

$$
\left|\nabla K_{t}(x, y)\right| \leq c t^{-1 / 2} V\left(y, t^{1 / 2}\right)^{-1}
$$

for all $t>0, x, y \in M$.

We also consider an infinite family of conditions indexed by $p \in[1, \infty]$.

Condition $(\mathrm{III})_{p}$. There is $c>0$ such that

$$
\left\|\nabla K_{t}(x, \cdot)\right\|_{p} \leq c t^{-1 / 2} V\left(x, t^{1 / 2}\right)^{-(1-(1 / p))}
$$

for all $t>0$ and $x \in M$.

Observe that Condition (III) $)_{\infty}$ says exactly that

$$
\left|\nabla K_{t}(x, y)\right| \leq c t^{-1 / 2} V\left(x, t^{1 / 2}\right)^{-1}
$$

for all $t>0, x, y \in M$, which is a variation of Condition (II). Also note that Condition (III) $)_{1}$ is equivalent to an operator norm estimate

$$
\left\|\nabla S_{t}\right\|_{\infty \rightarrow \infty} \leq c t^{-1 / 2}
$$

for all $t>0$, where in general $\|\cdot\|_{p_{1} \rightarrow p_{2}}$ denotes the norm of a bounded operator from $L^{p_{1}}$ to $L^{p_{2}}$.

We next state our main theorem.

Theorem 1.1. Assume that (1.1) and (1.2) hold on M. Then Conditions (I), (II), and (III) are equivalent, for each $p \in[1, \infty]$. Moreover, if these conditions hold then estimate (1.3) holds for each $b \in(0,1 / 4)$ with some $c=c(b)>0$ depending on $b$.

Some known examples of $M$ where (1.1), (1.2), and Condition (I) hold, are (i) manifolds of nonnegative Ricci curvature (see Li and Yau [8]) and (ii) Galois covering 
manifolds of compact manifolds whose deck transformation group is of polynomial growth (see [4]). Analogous estimates are known for subelliptic sublaplacians on Lie groups of polynomial growth (see, e.g., [13]). There are also simple examples of manifolds satisfying (1.1) and (1.2) but not Condition (I): see for example [3, Section 5], where $M$ is formed by glueing two copies of $\mathbb{R}^{n}$.

The main tool used in proving Theorem 1.1 is the finite speed propagation of the wave equation associated with $H$, and our proof is influenced by arguments of Sikora [10] and ter Elst, Robinson and Sikora [12, Section 2].

As an application of Theorem 1.1, we get a result about stability of Condition (I) under certain mappings. Let $\pi: M \rightarrow M_{1}$ be a smooth, surjective mapping of Riemannian manifolds which is a submersion, that is, the differential $\pi_{*}: T_{x} M \rightarrow T_{\pi(x)} M_{1}$ is surjective for all $x \in M$. Let us say that $\pi$ preserves Laplacians if $H(f \circ \pi)=\left(H_{1} f\right) \circ \pi$ for all $f \in C_{c}^{\infty}\left(M_{1}\right)$ where $H, H_{1}$ are the Laplace operators on $M, M_{1}$, respectively.

Theorem 1.2. Let $\pi: M \rightarrow M_{1}$ be a surjective submersion as above, which preserves Laplacians. Suppose that (1.1), (1.2), and Condition (I) hold on M. Then (1.1), (1.2), and Condition (I) hold on $M_{1}$.

Important recent work [2] shows that Condition (I) (or Condition (II)), together with (1.1) and (1.2), is sufficient to obtain the boundedness of the Riesz transform operator $\nabla H^{-1 / 2}$ in $L^{p}(M)$ for all $1<p<\infty$. Applying this result in the context of Theorem 1.2 gives the following statement.

Corollary 1.3. Adopt the hypotheses of Theorem 1.2. Then the Riesz transform $\nabla H_{1}^{-1 / 2}$ is bounded in $L^{p}\left(M_{1}\right)$ for $1<p<\infty$.

In Section 3 we apply Theorem 1.2 and Corollary 1.3 in some specific cases, namely, co-compact covering manifolds or homogeneous spaces of Lie groups of polynomial growth, to derive new results.

Finally, let us describe a generalization of Theorem 1.1 to a wider context than the above Riemannian setting. Suppose $(M, d)$ is a metric space, endowed with a Borel measure $\mu$ such that the doubling property (1.1) holds with $V(x, r):=\mu(\{y \in M: d(x, y)<$ $r$ ) ). Let $H$ be a nonnegative selfadjoint operator in $L^{2}=L^{2}(M ; \mu)$, such that the semigroup $S_{t}=e^{-t H}, t>0$, has a continuous kernel $K_{t}: M \times M \rightarrow \mathbb{R}$ satisfying Gaussian estimates (1.2). Assume that the wave equation associated with $H$ has propagation of finite speed 1 , that is, $\operatorname{supp}\left(\cos \left(t H^{1 / 2}\right) f\right) \subseteq \operatorname{supp}_{t} f$ for any $f \in L^{2}, t>0$. Here, supp $f$ denotes the support of $f$ and $\operatorname{supp}_{t} f:=\{x \in M: d(x, \operatorname{supp} f) \leq t\}$.

Next, let $E$ be a continuous vector bundle over $M$ and let $(\cdot, \cdot)_{x}$ be a positive-definite inner product on the fibres $E_{x}, x \in M$, which varies continuously with $x$. Using the length $|v|_{x}:=(v, v)_{x}^{1 / 2}, v \in E_{x}$, we may form the spaces $L^{p}(E)=L^{p}(E ; \mu)$ of measurable, $p$-integrable sections of $E$. Let $\nabla$ denote a linear operator mapping a dense subspace of $L^{2}(M)$ into $L^{2}(E)$, such that $\nabla$ is local, that is, $\operatorname{supp}(\nabla f) \subseteq \operatorname{supp} f$ for any $f \in D(\nabla)$.

Then Theorem 1.1 remains valid under these more general assumptions. Moreover, one could replace the factor $t^{-1 / 2}$ occurring in Conditions (I), (II), (III) $p$ with $t^{-\alpha}$ for any fixed constant $\alpha>0$. To prove these assertions requires only trivial modifications in the proof of Theorem 1.1. Note that the above general setting is essentially that considered in [10]. 
4 Some remarks on gradient estimates for heat kernels

\section{Proofs}

For the proof of Theorem 1.1 we first collect a number of preliminary results. In general, $c, c^{\prime}, b$ and so on denote positive constants whose value may change from line to line when convenient. The integral kernel of a linear operator $A$ acting on functions on $M$ is denoted by $K_{A}=K_{A}(x, y)$ (in particular, $K_{t}=K_{S_{t}}=K_{e^{-t H}}$ for $t>0$ ).

A standard consequence of (1.1) is that there is a constant $D>0$ such that

$$
V(x, \gamma r) \leq c(1+\gamma)^{D} V(x, r)
$$

for all $r>0, y>0$ and $x \in M$. Observing that $B\left(y, t^{1 / 2}\right) \subseteq B\left(x, t^{1 / 2}+d(x, y)\right)$ and applying (2.1), we deduce that

$$
V\left(x, t^{1 / 2}\right) \geq c^{\prime}\left(1+d(x, y) t^{-1 / 2}\right)^{-D} V\left(y, t^{1 / 2}\right)
$$

for all $t>0$ and $x, y \in M$. This bound allows one, for example, to replace $V\left(y, t^{1 / 2}\right)$ with $V\left(x, t^{1 / 2}\right)$ in (1.2) or in Condition (I). Another straightforward consequence of (1.1) is that for any $b>0$ there is a $c=c(b)>0$ such that

$$
\int_{M} d x e^{-b d(x, y)^{2} / t} \leq c V\left(y, t^{1 / 2}\right)
$$

for all $t>0$ and $y \in M$.

Lemma 2.1. Let $p \in[1, \infty]$. Given $m \in \mathbb{N}$ with $m>2^{-1} D\left(1-p^{-1}\right)$, there exists $c=c(m)>$ 0 such that

$$
\left\|K_{(I+t H)^{-m}}(\cdot, y)\right\|_{p}=\left\|K_{(I+t H)^{-m}}(y, \cdot)\right\|_{p} \leq c V\left(y, t^{1 / 2}\right)^{-(1-(1 / p))}
$$

for all $t>0$ and $y \in M$.

Proof. Note that

$$
(I+t H)^{-m}=\Gamma(m)^{-1} \int_{0}^{\infty} d s e^{-s} s^{m-1} S_{s t} .
$$

Moreover, (1.2) and (2.3) yield a bound $\left\|K_{u}(\cdot, y)\right\|_{p} \leq V\left(y, u^{1 / 2}\right)^{-(1-(1 / p))}$ for all $u>0$, $y \in M$. Hence using (2.1), we have

$$
\begin{aligned}
\| K_{(I+t H)^{-m}(\cdot, y) \|_{p}} & \leq c^{\prime} \int_{0}^{\infty} e^{-s} s^{m-1} V\left(y,(s t)^{1 / 2}\right)^{-(1-(1 / p))} \\
& \leq c V\left(y, t^{1 / 2}\right)^{-(1-(1 / p))} \int_{0}^{\infty} e^{-s} s^{m-1}\left(1+s^{-1}\right)^{(D / 2)(1-(1 / p))} \\
& =c^{\prime} V\left(y, t^{1 / 2}\right)^{-(1-(1 / p))}
\end{aligned}
$$

where the last integral converges because $m>(D / 2)(1-(1 / p))$.

Fix a nondecreasing function $\psi \in C^{\infty}(\mathbb{R})$ with $\psi(x)=0$ for all $x \leq-1$ and $\psi(x)=1$ for all $x \geq-2^{-1}$, and for each $\rho>1$ define $F_{\rho} \in C^{\infty}(\mathbb{R})$ by

$$
F_{\rho}(x)=\psi(\rho(|x|-\rho))(4 \pi)^{-1 / 2} e^{-x^{2} / 4} .
$$


Consider the Fourier transform $\widehat{F_{\rho}}$ of $F_{\rho}$. For each $t>0, \rho>1$ and $m \in \mathbb{N}_{0}=\{0,1,2, \ldots\}$ define the operator

$$
S_{t}^{(\rho, m)}=(I+t H)^{m} \widehat{F_{\rho}}\left((t H)^{1 / 2}\right)
$$

which is selfadjoint in $L^{2}(M)$, and denote by $K_{t}^{(\rho, m)}(x, y)=K_{t}^{(\rho, m)}(y, x)$ its integral kernel. The next lemma, which follows from results of [10], is the place where we use finite speed propagation of the wave equation associated with $H$.

Lemma 2.2. One has $K_{t}^{(\rho, 0)}(x, y)=K_{t}(x, y)$ for all $t>0, \rho>1$ and $x, y \in M$ such that $d(x, y)>\rho t^{1 / 2}$.

For any $m \in \mathbb{N}_{0}$ and $\omega \in(0,1 / 4)$, there exists $c=c(m, \omega)>0$ such that

$$
\left|\left(1+\lambda^{2}\right)^{m} \widehat{F_{\rho}}(\lambda)\right| \leq c e^{-\omega \rho^{2}}
$$

for all $\rho>1$ and $\lambda \geq 0$.

Indeed, the first statement of Lemma 2.2 follows in the same way as [10, equation (5.3)], while (2.9) is a cruder version of [10, inequality (5.2)].

We now establish useful pointwise bounds on the kernels $K_{t}^{(\rho, m)}$.

Lemma 2.3. Given $m \in \mathbb{N}_{0}, \omega \in(0,1 / 4)$, there exist positive constants $c=c(m, \omega), b=$ $b(m, \omega)$ such that

$$
\left|K_{t}^{(\rho, m)}(x, y)\right| \leq c e^{-\omega \rho^{2}} V\left(y, t^{1 / 2}\right)^{-1} e^{-b d(x, y)^{2} / t}
$$

for all $\rho>1, t>0$ and $x, y \in M$.

Proof. In this proof, we fix an $\omega \in(0,1 / 4)$ and denote by $c_{m}$ constants depending on $m$. By the spectral theorem for $H$, and (2.9), for each $m \in \mathbb{N}_{0}$ one has

$$
\left\|S_{t}^{(\rho, m)}\right\|_{2 \rightarrow 2} \leq c_{m} e^{-\omega \rho^{2}}
$$

for all $t>0$ and $\rho>1$. Next, writing

$$
K_{t}^{(\rho, m)}(x, y)=\int_{M} d z K_{t}^{(\rho, m+n)}(x, z) K_{(I+t H)^{-n}}(z, y)
$$

for $n \in \mathbb{N}$, it follows that

$$
K_{t}^{(\rho, m)}(\cdot, y)=S_{t}^{(\rho, m+n)}\left(K_{(I+t H)^{-n}}(\cdot, y)\right)
$$

Hence by fixing $n$ sufficiently large and applying Lemma 2.1, one finds

$$
\begin{aligned}
\left\|K_{t}^{(\rho, m)}(\cdot, y)\right\|_{2} & \leq\left\|S_{t}^{(\rho, m+n)}\right\|_{2 \rightarrow 2}\left\|K_{(I+t H)^{-n}}(\cdot, y)\right\|_{2} \\
& \leq c_{m} e^{-\omega \rho^{2}} V\left(y, t^{1 / 2}\right)^{-1 / 2}
\end{aligned}
$$


6 Some remarks on gradient estimates for heat kernels

for all $t>0, \rho>1$, and $y \in M$. Replacing $m$ by $m+n$ in this estimate, and applying Hölder's inequality in (2.12), yields

$$
\begin{aligned}
\left|K_{t}^{(\rho, m)}(x, y)\right| & \leq\left\|K_{t}^{(\rho, m+n)}(x, \cdot)\right\|_{2}\left\|K_{(I+t H)^{-n}}(\cdot, y)\right\|_{2} \\
& \leq c_{m} e^{-\omega \rho^{2}} V\left(x, t^{1 / 2}\right)^{-1 / 2} V\left(y, t^{1 / 2}\right)^{-1 / 2}
\end{aligned}
$$

for all $t>0, \rho>1$ and $x, y \in M$. Now, in case $d(x, y) \leq \rho t^{1 / 2}$, this estimate yields for any $\varepsilon \in(0, \omega)$ that

$$
\left|K_{t}^{(\rho, m)}(x, y)\right| \leq c_{m} e^{-(\omega-\varepsilon) \rho^{2}} V\left(x, t^{1 / 2}\right)^{-1 / 2} V\left(y, t^{1 / 2}\right)^{-1 / 2} e^{-\varepsilon d(x, y)^{2} / t} .
$$

In the alternative case where $d(x, y)>\rho t^{1 / 2}$, Lemma 2.2 implies that

$$
K_{t}^{(\rho, m)}(x, y)=\left((I+t H)^{m}\right)_{x} K_{t}(x, y)=K_{t}^{(s, m)}(x, y)
$$

for any $s \in\left(\rho, d(x, y) t^{-1 / 2}\right)$. Then

$$
\begin{aligned}
\left|K_{t}^{(\rho, m)}(x, y)\right| & \leq c_{m} e^{-\omega s^{2}} V\left(x, t^{1 / 2}\right)^{-1 / 2} V\left(y, t^{1 / 2}\right)^{-1 / 2} \\
& \leq c_{m} e^{-(\omega-\varepsilon) \rho^{2}} V\left(x, t^{1 / 2}\right)^{-1 / 2} V\left(y, t^{1 / 2}\right)^{-1 / 2} e^{-\varepsilon s^{2}}
\end{aligned}
$$

for any $\varepsilon \in(0, \omega)$ and $s \in\left(\rho, d(x, y) t^{-1 / 2}\right)$, and hence also for $s=d(x, y) t^{-1 / 2}$. Combining the two cases above and using (2.2), for any $\varepsilon \in(0, \omega)$ we find an estimate

$$
\left|K_{t}^{(\rho, m)}(x, y)\right| \leq c_{m, \varepsilon} e^{-(\omega-\varepsilon) \rho^{2}} V\left(y, t^{1 / 2}\right)^{-1} e^{-2^{-1} \varepsilon d(x, y)^{2} / t}
$$

for all $t>0, \rho>1$ and $x, y \in M$. The lemma follows.

Remark 2.4. By similar but simpler arguments to those of Lemma 2.3, one may prove the estimates, for each $m \in \mathbb{N}_{0}$ and $\omega \in(0,1 / 4)$,

$$
\left|\left((I+t H)^{m}\right)_{x} K_{t}(x, y)\right| \leq c(m, \omega) V\left(y, t^{1 / 2}\right)^{-1} e^{-\omega d(x, y)^{2} / t}
$$

for all $t>0, x, y \in M$. Since $\left(H^{m}\right)_{x} K_{t}(x, y)=(-1)^{m}(\partial / \partial t)^{m} K_{t}(x, y)$, the latter estimates amount to estimates on time derivatives of $K_{t}$, which are well known consequences of (1.2) (see, e.g., [6] and its references).

Integrating the estimate of Lemma 2.3 using (2.3) easily yields the following bounds. Corollary 2.5. Given $m \in \mathbb{N}_{0}, \omega \in(0,1 / 4)$ and $p \in[1, \infty]$, there is a $c>0$ such that

$$
\left\|K_{t}^{(\rho, m)}(\cdot, y)\right\|_{p}=\left\|K_{t}^{(\rho, m)}(y, \cdot)\right\|_{p} \leq c e^{-\omega \rho^{2}} V\left(y, t^{1 / 2}\right)^{-(1-(1 / p))}
$$

for all $t>0, \rho>1$ and $y \in M$.

We are ready to prove Theorem 1.1. Condition (I) implies Condition (II) trivially, and Condition (I) implies Condition (III) $)_{p}$ by integration using (2.3). 
Next, let us assume Condition (III) $p$ for a fixed $p \in[1, \infty]$, and deduce Condition (I). First consider the case where $d(x, y)>t^{1 / 2}$. For any $\rho \in\left(1, d(x, y) t^{-1 / 2}\right)$, by Lemma 2.2

$$
\nabla K_{t}(x, y)=\nabla K_{t}^{(\rho, 0)}(x, y)=\int_{M} d z\left(\nabla K_{(I+t H)^{-m}}(x, z)\right) K_{t}^{(\rho, m)}(z, y) .
$$

From Condition (III) $p$ we may argue as in the proof of Lemma 2.1, to deduce for some fixed $m \in \mathbb{N}$ that

$$
\left\|\nabla K_{(I+t H)^{-m}}(x, \cdot)\right\|_{p} \leq c t^{-1 / 2} V\left(x, t^{1 / 2}\right)^{-(1-(1 / p))}
$$

for all $t>0, x \in M$. Fix $\omega \in(0,1 / 4)$ and let $1 / p^{\prime}=1-(1 / p)$. By Hölder's inequality and Corollary 2.5,

$$
\begin{aligned}
\left|\nabla K_{t}(x, y)\right| & \leq\left\|\nabla K_{(I+t H)^{-m}}(x, \cdot)\right\|_{p}\left\|K_{t}^{(\rho, m)}(\cdot, y)\right\|_{p^{\prime}} \\
& \leq c t^{-1 / 2} V\left(x, t^{1 / 2}\right)^{-(1-(1 / p))} V\left(y, t^{1 / 2}\right)^{-1 / p} e^{-\omega \rho^{2}}
\end{aligned}
$$

whenever $\rho \in\left(1, d(x, y) t^{-1 / 2}\right)$; this estimate then holds with $\rho=d(x, y) t^{-1 / 2}$.

In case $d(x, y) \leq t^{1 / 2}$, we write $\nabla K_{t}(x, y)=\int_{M} d z\left(\nabla K_{t / 2}(x, z)\right) K_{t / 2}(z, y)$ so that

$$
\begin{aligned}
\left|\nabla K_{t}(x, y)\right| & \leq\left\|\nabla K_{t / 2}(x, \cdot)\right\|_{p}\left\|K_{t / 2}(\cdot, y)\right\|_{p^{\prime}} \\
& \leq c t^{-1 / 2} V\left(x, t^{1 / 2}\right)^{-(1-(1 / p))} V\left(y, t^{1 / 2}\right)^{-1 / p} .
\end{aligned}
$$

Combining the two cases above, and using (2.2), we get Condition (I).

Finally, we show that Condition (II) implies Condition (I). From Condition (II), by arguing as in Lemma 2.1, we get for some fixed $m \in \mathbb{N}$ a bound

$$
\left|\nabla K_{(I+t H)^{-m}}(x, y)\right| \leq c t^{-1 / 2} V\left(y, t^{1 / 2}\right)^{-1}
$$

for all $t>0$ and $x, y \in M$. When $d(x, y)>t^{1 / 2}$, we insert this estimate in (2.22) and use Lemma 2.3 with some $\omega \in(0,1 / 4)$, to get

$$
\begin{aligned}
\left|\nabla K_{t}(x, y)\right| & \leq c t^{-1 / 2} \int_{M} d z V\left(z, t^{1 / 2}\right)^{-1}\left|K_{t}^{(\rho, m)}(z, y)\right| \\
& \leq c^{\prime} t^{-1 / 2} e^{-\omega \rho^{2}} \int_{M} d z V\left(z, t^{1 / 2}\right)^{-1} V\left(y, t^{1 / 2}\right)^{-1} e^{-b d(z, y)^{2} / t} \\
& \leq c^{\prime \prime} t^{-1 / 2} e^{-\omega \rho^{2}} V\left(y, t^{1 / 2}\right)^{-1}
\end{aligned}
$$

for all $\rho \in\left(1, d(x, y) t^{-1 / 2}\right)$. Here, the last line is a straightforward consequence of (2.2) and (2.3). This establishes the estimate of Condition (I) in case $d(x, y)>t^{1 / 2}$. The case $d(x, y) \leq t^{1 / 2}$ is immediate from Condition (II). This finishes the proof that Conditions (I), (II), (III $)_{p}$ are equivalent. The last statement of Theorem 1.1 follows easily from the above arguments since $\omega \in(0,1 / 4)$ can be chosen arbitrarily. The proof of Theorem 1.1 is complete.

Let us prove Theorem 1.2. First, work of Saloff-Coste [9] shows that (1.1) and (1.2) hold on $M_{1}$. Indeed, it is well known that (1.1), (1.2), and Condition (I) on $M$ imply a 
global parabolic Harnack inequality on $M$, and since $\pi: M \rightarrow M_{1}$ preserves Laplacians a Harnack inequality on $M_{1}$ easily follows: see [9, page 36]. But the Harnack inequality implies (1.1) and (1.2) on $M_{1}$, again according to [9].

To obtain Condition (I) on $M_{1}$, consider the semigroups $S_{t}=e^{-t H}$ on $M$ and $S_{t}^{(1)}=$ $e^{-t H_{1}}$ on $M_{1}$. Since $\pi$ preserves Laplacians, standard semigroup algorithms yield that

$$
S_{t}(f \circ \pi)=\left(S_{t}^{(1)} f\right) \circ \pi
$$

for all $f \in L^{\infty}\left(M_{1}\right)$. Moreover, the pointwise lengths of the gradients on $M$ and $M_{1}$ are related by

$$
|\nabla(f \circ \pi)|=|\nabla f| \circ \pi
$$

for all $f \in C^{\infty}\left(M_{1}\right)$. This follows immediately from the standard identity

$$
|\nabla \tilde{f}|^{2}=-2^{-1} H\left(\tilde{f}^{2}\right)+\tilde{f}(H \tilde{f})
$$

for $\tilde{f} \in C^{\infty}(M)$ and the analogous identity on $M_{1}$, because $\pi$ preserves Laplacians. Combining these observations, and using the equivalence of Condition (I) with (1.7) given by Theorem 1.1, we obtain

$$
\begin{aligned}
\left\|\left|\nabla S_{t}^{(1)} f\right|\right\|_{L^{\infty}\left(M_{1}\right)} & =\left\|\left|\nabla S_{t}^{(1)} f\right| \circ \pi\right\|_{L^{\infty}(M)}=\left\|\left|\nabla S_{t}(f \circ \pi)\right|\right\|_{L^{\infty}(M)} \\
& \leq c t^{-1 / 2}\|f \circ \pi\|_{L^{\infty}(M)}=c t^{-1 / 2}\|f\|_{L^{\infty}\left(M_{1}\right)}
\end{aligned}
$$

for all $t>0$ and $f \in L^{\infty}\left(M_{1}\right)$. This establishes estimate (1.7) on $M_{1}$. Then Theorem 1.2 follows by once again applying Theorem 1.1 .

\section{Examples}

We give two examples which illustrate our results.

Example (I). Let $M$ be a Riemannian manifold which is a Galois covering manifold of a compact Riemannian manifold $M_{0}$. In other words, there is a discrete, finitely generated group $G$ of isometries acting properly and freely on $M$, such that the quotient $M_{0}=M / G$ is compact. Let us also assume that the group $G$ has polynomial volume growth (see, e.g., [13, Sections VI.2 and X.3] for background). In this situation it is well known that assumptions (1.1) and (1.2) hold on $M$ : see [9, Page 36] or [7, Section 7.6] for instance. The author [4, Theorem 1.1] proved Condition (I) on $M$.

Let $G_{1}$ be any (possibly nonnormal) subgroup of $G$, and form the quotient manifold $M_{1}=M / G_{1}$ with Riemannian metric induced by the metric of $M$. Then $M_{1}$ is a covering (though in general not a Galois covering) of $M_{0}$. The natural map $\pi: M \rightarrow M_{1}$ is a covering map and a local isometry, and hence preserves Laplacians. Thus combining the results mentioned above with Theorem 1.2, we obtain the following statement. 
Corollary 3.1. Let $M_{1}=M / G_{1}$ be as above. Then Condition (I) holds on $M_{1}$.

Note that (1.1) and (1.2) and also a parabolic Harnack inequality were already known on $M_{1}$ : see [9, Page 36]. However, Condition (I) seems to be new in this setting. Also, Corollary 1.3 applies to give boundedness of the Riesz transform on $M_{1}$.

Example (II). In this example we consider certain subelliptic operators in place of the Laplace operator, but the principles of Theorems 1.1 and 1.2 still apply (compare the remarks at the end of Section 1 ).

Let $G$ be a Lie group of polynomial volume growth and consider a sublaplacian

$$
H=-\sum_{i=1}^{d^{\prime}} A_{i}^{2}
$$

on $G$ (for background, see [13] and references therein). Here, $A_{1}, \ldots, A_{d^{\prime}}$ is a list of right invariant vector fields on $G$ which generates algebraically the Lie algebra $\mathfrak{g}$ of $G$. The operator $H$ generates a symmetric submarkovian semigroup $e^{-t H}$ in $L^{p}=L^{p}(G ; d g), 1 \leq$ $p \leq \infty$, where $d g$ is a fixed Haar measure for $G$. Associated with $H$ is a subelliptic gradient $\nabla f:=\left(A_{1} f, \ldots, A_{d^{\prime}} f\right), f \in C^{\infty}(G)$, and a distance $d$ with

$$
d(x, y)=\sup \left\{\psi(x)-\psi(y): \psi \in C^{\infty}(G),|\nabla \psi| \leq 1\right\} .
$$

It is well known that estimates (1.1) and (1.2) and Condition (I) hold on $G$ (see [13, Section VIII.2]), and the finite speed propagation property holds for $H$. In the special case where $A_{1}, \ldots, A_{d^{\prime}}$ form a vector space basis for $\mathfrak{g}$, one easily sees that $H$ is the Laplace operator associated with a right invariant Riemannian metric on $G$.

Now let $G_{1}$ be any closed subgroup of $G$, and form the smooth manifold $M_{1}=G / G_{1}$ consisting of all left cosets $g G_{1}, g \in G$, of $G_{1}$. Consider the projection $\pi: G \rightarrow M_{1}, \pi(g)=$ $g G_{1}$, with differential $\pi_{*}: T G \rightarrow T M_{1}$. Since $A_{i}$ is right invariant, one easily checks that $\pi_{*}\left(\left.A_{i}\right|_{g}\right)=\pi_{*}\left(\left.A_{i}\right|_{g g_{1}}\right) \in T_{\pi(g)} M_{1}$ for all $g \in G$ and $g_{1} \in G_{1}$. Therefore $B_{i}:=\pi_{*}\left(A_{i}\right)$ are well-defined vector fields on $M_{1}$, and we may form the subelliptic operator

$$
H_{1}=-\sum_{i=1}^{d^{\prime}} B_{i}^{2}
$$

on $M_{1}$. Then $H(f \circ \pi)=\left(H_{1} f\right) \circ \pi$ for all $f \in C^{\infty}\left(M_{1}\right)$.

To $H_{1}$ we associate a gradient $\nabla f=\left(B_{1} f, \ldots, B_{d^{\prime}} f\right), f \in C^{\infty}\left(M_{1}\right)$, and a distance $d$ on $M_{1}$ by a formula analogous to (3.2). Fix a positive Borel measure $\mu$ on $M_{1}$ which is invariant under the standard action $g \cdot\left(h G_{1}\right):=g h G_{1}, g, h \in G$, of $G$ on $M_{1}$, and consider the spaces $L^{p}\left(M_{1}\right)=L^{p}\left(M_{1} ; \mu\right)$ (the existence of such a measure follows from the unimodularity of $G$ and $G_{1}$ : see, e.g., [5, Theorem 2.49]). One has $\left(H_{1} f_{1}, f_{1}\right) \geq 0,\left(H_{1} f_{1}, f_{2}\right)=$ $\left(f_{1}, H_{1} f_{2}\right)$ for all $f_{1}, f_{2} \in C_{c}^{\infty}\left(M_{1}\right)$, and the Friedrichs extension of $H_{1}$ is a nonnegative selfadjoint operator in $L^{2}\left(M_{1}\right)$ which generates a symmetric submarkovian semigroup $e^{-t H_{1}}$.

We remark that, according to general theory of partial differential operators on Lie groups (see [11] for instance), one can identify $H_{1}=d U(H)$ where $U$ is the unitary representation of $G$ in $L^{2}\left(M_{1}\right)$ with $(U(g) f)\left(h G_{1}\right)=f\left(g^{-1} h G_{1}\right), g, h \in G$. 
The proof of Theorem 1.2 goes through in this situation, and therefore (1.1) and (1.2) and Condition (I) hold on $M_{1}$. To our knowledge, Condition (I) is new in this setting.

Finally, the results of [2] apply to give the boundedness of the Riesz transform $\nabla H_{1}^{-1 / 2}$ in $L^{p}\left(M_{1}\right), 1<p<\infty$. This seems to be a new result (the boundedness of the Riesz transform on the group $G$ was first proved in [1]).

\section{Acknowledgment}

This work was carried out with financial support from the Australian Research Council (ARC) Centre of Excellence for Mathematics and Statistics of Complex Systems (MAS$\mathrm{COS})$.

\section{References}

[1] G. Alexopoulos, An application of homogenization theory to harmonic analysis: Harnack inequalities and Riesz transforms on Lie groups of polynomial growth, Canadian Journal of Mathematics. Journal Canadien de Mathématiques 44 (1992), no. 4, 691-727.

[2] P. Auscher, T. Coulhon, X. T. Duong, and S. Hofmann, Riesz transform on manifolds and heat kernel regularity, Annales Scientifiques de École Normale Supérieure (4) 37 (2004), no. 6, 911957.

[3] T. Coulhon and X. T. Duong, Riesz transforms for $1 \leqslant p \leqslant 2$, Transactions of the American Mathematical Society 351 (1999), no. 3, 1151-1169.

[4] N. Dungey, Heat kernel estimates and Riesz transforms on some Riemannian covering manifolds, Mathematische Zeitschrift 247 (2004), no. 4, 765-794.

[5] G. B. Folland, A Course in Abstract Harmonic Analysis, Studies in Advanced Mathematics, CRC Press, Florida, 1995.

[6] A. Grigor'yan, Upper bounds of derivatives of the heat kernel on an arbitrary complete manifold, Journal of Functional Analysis 127 (1995), no. 2, 363-389.

[7] __ Estimates of heat kernels on Riemannian manifolds, Spectral Theory and Geometry (Edinburgh, 1998) (E. B. Davies and Y. Safarov, eds.), London Math. Soc. Lecture Note Ser., vol. 273, Cambridge University Press, Cambridge, 1999, pp. 140-225.

[8] P. Li and S.-T. Yau, On the parabolic kernel of the Schrödinger operator, Acta Mathematica 156 (1986), no. 3-4, 153-201.

[9] L. Saloff-Coste, A note on Poincaré, Sobolev, and Harnack inequalities, International Mathematics Research Notices 1992 (1992), no. 2, 27-38.

[10] A. Sikora, Riesz transform, Gaussian bounds and the method of wave equation, Mathematische Zeitschrift 247 (2004), no. 3, 643-662.

[11] A. F. M. ter Elst and D. W. Robinson, Weighted subcoercive operators on Lie groups, Journal of Functional Analysis 157 (1998), no. 1, 88-163.

[12] A. F. M. ter Elst, D. W. Robinson, and A. Sikora, Riesz transforms and Lie groups of polynomial growth, Journal of Functional Analysis 162 (1999), no. 1, 14-51.

[13] N. T. Varopoulos, L. Saloff-Coste, and T. Coulhon, Analysis and Geometry on Groups, Cambridge Tracts in Mathematics, vol. 100, Cambridge University Press, Cambridge, 1992.

Nick Dungey: School of Mathematics, The University of New South Wales, Sydney 2052, Australia E-mail address: dungey@maths.unsw.edu.au 


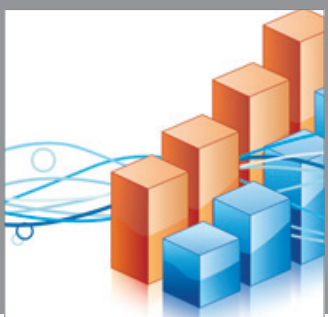

Advances in

Operations Research

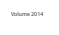

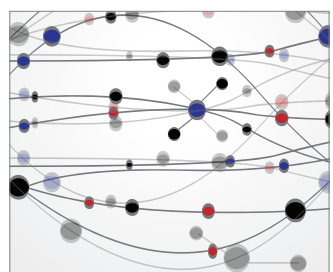

\section{The Scientific} World Journal
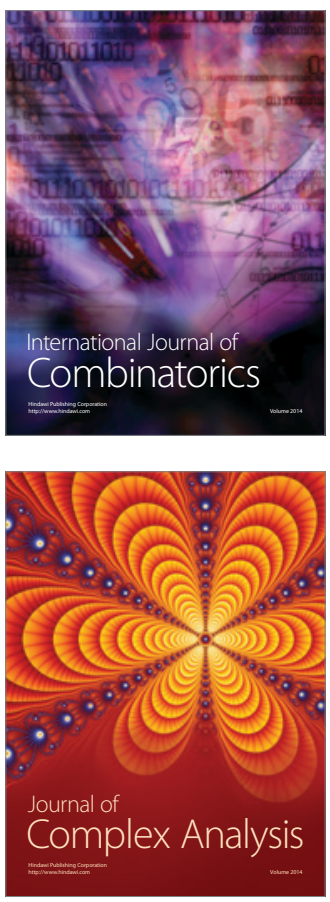

International Journal of

Mathematics and

Mathematical

Sciences
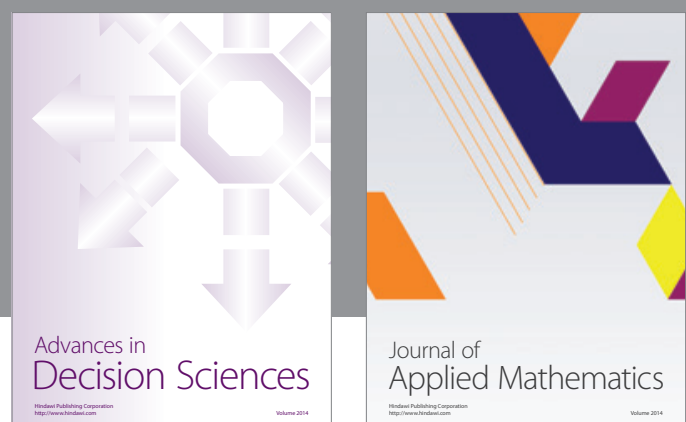

Journal of

Applied Mathematics
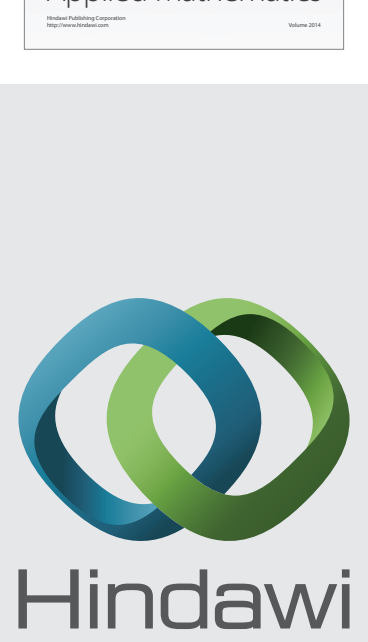

Submit your manuscripts at http://www.hindawi.com
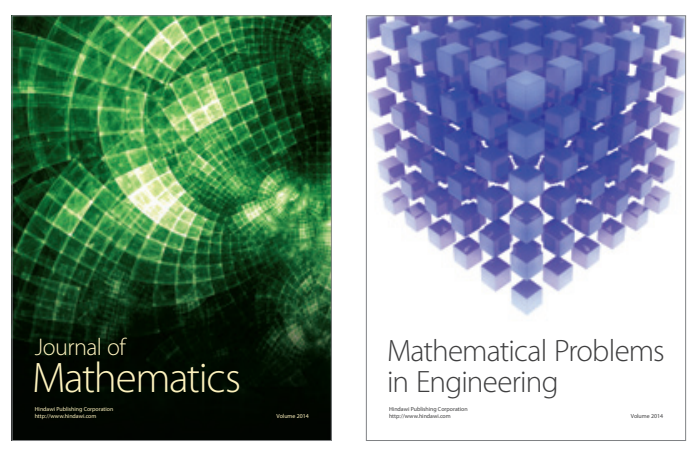

Mathematical Problems in Engineering
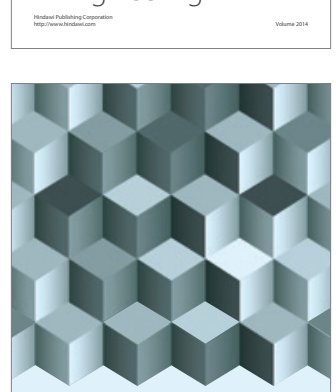

Journal of

Function Spaces
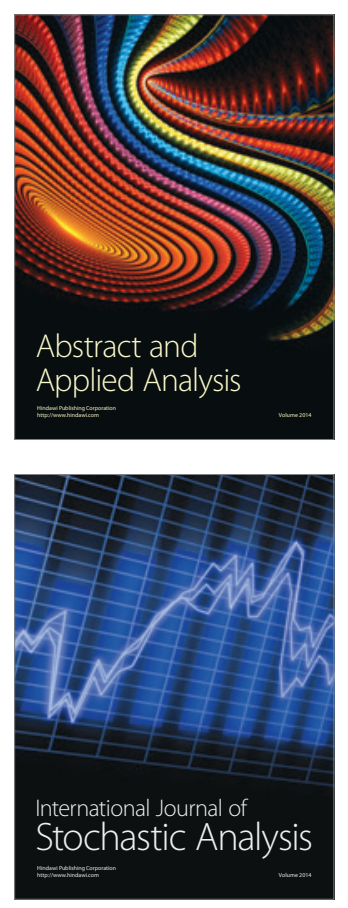

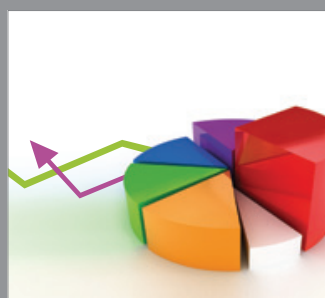

ournal of

Probability and Statistics

Promensencen
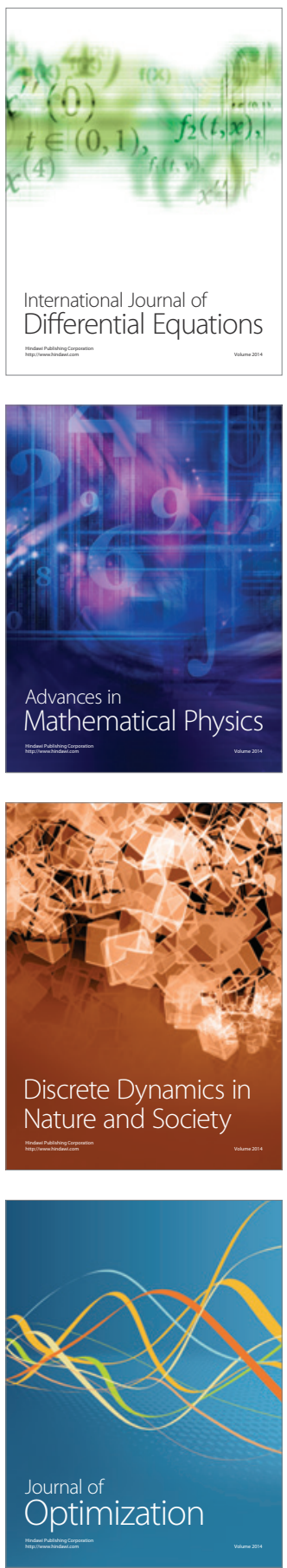